\title{
FAMILIAL PAPILLARY THYROID CARCINOMA: A CASE SERIES
}

\author{
Ahmed Imran Siddiqi \\ Department of Internal Medicine, Shaukat Khanum Memorial Cancer Hospital and Research Center, Lahore, \\ Pakistan
}

Received: 09 March 2017 / Accepted: 15 March 2017

\begin{abstract}
The natural history and inheritance of familial papillary thyroid carcinoma (FPTC) remain unclear. A specific genetic defect responsible for this condition and its inheritance is yet to be established. The penetrance, mode of inheritance and treatment of this familial thyroid condition are different from other familial thyroid conditions (familial medullary thyroid cancer). Presented here is a family of four sisters in which three developed multifocal PTC. A 47-year-old lady presented with incidental finding of multinodular goitre and normal thyroid function. She had three other sisters who were diagnosed with PTC at age 46, 48 and 49 years. Detailed clinical history of her sisters was gathered from them over telephone as they all moved to different parts of world in their twenties. Literature review was carried out to find similar patients reported in literature. Environmental factors were less important in the development of PTC in this family. It was also interesting that all three were diagnosed with the condition at a very similar age. FPTC behaviour was not more aggressive than sporadic form of disease. There is not enough evidence in literature to suggest prophylactic thyroidectomy in relatives of patients with PTC. Since the exact inheritance pattern of this condition is not known, detailed family history is important in patients with thyroid disorders to identify high-risk patients. Genetic factors are much more important than environmental factors in the development of this condition. The first-degree relatives should be assessed clinically for further investigations if two individuals in the same family develop the condition at similar age.
\end{abstract}

Key words: Familial follicular thyroid cancer, familial papillary thyroid cancer, papillary thyroid cancer

\section{Introduction}

Thyroid cancer is the most common endocrine malignancy and demonstrated approximately two and a half-fold increase from 1973 to 2002. ${ }^{[1]}$ This increase in incidence is sometimes put down to early detection of pathology due to improvement in health-care facilities as the mortality rates remain the same over the years. ${ }^{[2]}$ Relative risk of familial primary cancer in thyroid gland is between 5- and 10.4-fold. This turns out to be the highest relative risk of familial cancer compared with all other organs. ${ }^{[3]}$ Papillary thyroid carcinoma (PTC) constitutes $80 \%$ of all thyroid cancers (1). About $5-15 \%$ of these PTC cases $^{[4]}$ are believed to be familial, often termed as familial PTC (FPTC).

Correspondence: Dr. Ahmed Imran Siddiqi, Department of Internal Medicine, Shaukat Khanum Memorial Cancer Hospital and Research Centre, Lahore, Pakistan. Email: ahmedimran@skm.org.pk

\section{Clinical Cases}

\section{Case 1}

A 47-year-old lady presented with multinodular goitre. She was incidentally found to have multinodular goitre by her general practitioner during clinic appointment for upper respiratory tract infection. She denied any change in her voice, swallowing or breathing difficulties and no clinical features of hypo- or hyper-thyroidism. She currently smoked 10-15 cigarettes per day, drank 5 to 10 units of alcohol in a week and not allergic to any medication. She always lived in London and worked as an accountant. She did not take regular medications and not suffered from any chronic illness. To the best of her knowledge, neither her parents nor any other relatives had any thyroid-related problems except her three elder sisters and all of them had PTC. All of them were diagnosed between the ages of 45 and 50 years. She had two daughters and a son and 
none of them suffered from thyroid-related problems. On examination, she had multinodular goitre, no lymph nodes in neck and no signs of thyroid eye disease. She then had ultrasound (US) guided fine-needle aspirations (FNAs) of one nodule in each lobe of the thyroid gland, which were reported as Thy 2 . The nodules were selected on US and all the nodules were reported low risk by US criteria. The FNA was repeated 7 months later and once again reported Thy 2. Following discussion in MDT, it was decided to continue monitoring her thyroid nodules with US and clinical examination.

\section{Case 2}

Her eldest sister presented to this hospital about 7 years ago with difficulty in swallowing with a long-standing multinodular goitre. She was 49 years old at that time. She did not notice change in size of her gland before presentation and her breathing and voice remained unaffected. Her barium swallow showed no obstruction at level of thyroid gland and flow volume loops were also normal. Her thyroid FNA of a right-sided $2.7 \times 2.1$ $\times 2.2 \mathrm{~mm}$ nodule was reported Thy 5 and histopathology report of biopsy specimen confirmed multifocal PTC following thyroidectomy. She lived in London till the age of 19 years and then moved to New York. She worked in the financial market till age 47 years and returned to London 2 years ago. She denied radiation or chemical exposure all her life. No metastases were identified, biopsy reported multifocal papillary carcinoma of thyroid and she remains disease free till now. She had two daughters and one of them had multinodular goitre.

\section{Case 3}

Her other elder sister lived in Italy since the age of 24. She worked as a fashion designer and had Thy 5 on her FNA there in Italy at age 46. Her biopsy specimen also showed multifocal papillary carcinoma of thyroid gland. She also had no exposure to radiations or chemicals and remains disease free after thyroidectomy. Her biopsy reported multifocal papillary carcinoma of thyroid. Her wound was complicated by keloid scar. Plastic surgery improved it, but it did not improve to her satisfaction. She had a son and a daughter. Her son had multinodular goitre and was receiving carbimazole for overactive thyroid gland. No more information about his medical condition could be sought.

\section{Case 4}

Her third sister also had very similar presentation and diagnosed at age 48 years. She had FNA about 10 years ago in Spain and further follow-up was not considered necessary in view of stable multinodular goitre and benign FNA. She worked as journalist and travelled a lot during her job. Her biopsy following total thyroidectomy reported $0.8 \mathrm{~mm}$ unifocal papillary carcinoma and no evidence of metastasis were detected. She remains disease free to date. She had no children.

\section{Discussion}

This family shows most of the features of FPTC reported in literature. Most of the patients suffering from FPTC are younger in age than non-FPTC patients and severity of FPTC increases in successive generations. Not only that FPTC patients frequently present with more aggressive tumours but also there is also a higher incidence of multifocal tumour deposits among such patients. Invasion of surrounding tissues including metastasis to lymph nodes is also more frequently found among patients of FPTC compared to non-FPTC patients. ${ }^{[4]}$ Like most other thyroid-related conditions, women make most of the patients of sporadic PTC. Men and children are usually less likely to be affected by FPTC. ${ }^{[5]}$ One should consider FPTC a possible diagnosis in a family when men and children outnumber women with PTC. In medical history of patients who present with thyroid nodules, one should always try to find out exact nature of condition if a male member of family suffered from thyroid disorder. All these features suggest a worse form of papillary cancer compared to sporadic form of this disease. There have been no clinical trials so far to suggest that these features could be used as criteria for screening other family members, but clinicians should keep a low threshold for investigating symptomatic family members of patients who present with above features.

Small sample size of patients with FPTC poses a challenge to devise a reliable screening or diagnostic criteria for this condition. One of the studies diagnosed FPTC when three or more family members had PTC. FPTC is very much a diagnosis of exclusion so none of these patients should be suffering from any of the other known syndromes or conditions associated with thyroid cancers. ${ }^{[4,5]}$ Genetic and environmental factors are believed to be the two most 
common and important factors in causation and inheritance of most of the cancers. In most of the patients, it is difficult to identify the factors responsible for this condition and its inheritance as they all share same genetic background and environmental factors. Not only that most cases of FPTC have a small pedigree but they also present with a variety of thyroid-related additional conditions. Most of these conditions are benign, but some may be contributing to the development of thyroid cancer. The presence of these additional conditions has made identification of genetic factors and the molecular alterations at the origin of the pathology very challenging. ${ }^{[5,6]}$

One should expect a certain set of specific genes inherited from one generation to another in these patients of FPTC. The inheritance pattern of this condition is still not clear. Genetic studies on patients with non-FPTC have managed to identify certain gene sequences associated with this condition. The point mutations of BRAF genes and rearrangements of chromosomes of the RET/PTC have been found in patients of sporadic PTC. Serine/threonine-protein kinases, a product of BRAF gene is involved in regulating MAP kinase/ERKs second messenger pathways. These signalling pathways influence functioning of cells including cell differentiation, cell division and secretion. Point mutations in BRAF are found in just under half(45\%) PTC cases. ${ }^{[7]}$ The involvement of this particular gene in a significant number of PTC patients has generated a lot of interest in this gene. Although BRAF is associated with a significant number of patients with non-FPTC, it is not associated with a sizeable number of patients with FPTC. This raises the question that is there separate genes responsible for non-FPTC and FPTC? This remains unanswered until we find the definitive genes responsible for each condition.

The other most commonly associated gene with nonFPTC was RET. Rearrangements of the RET are found in about $35 \%$ of sporadic PTC. The RET protooncogene is one of the receptor tyrosine kinases. These tyrosine kinase receptors are located on cell surface and transduce signals inside cell which affects cell growth and differentiation. ${ }^{[7]}$ This rearrangement of RET affects cell growth and differentiation. It has been associated with some other forms of cancers as well. Such a high incidence of these genetic mutations in BRAF and RET strongly suggests their association with non-FPTC; however, there should be effect of these mutations on germ line for these to be inheritable. In a study, germ line mutations of single-nucleotide polymorphisms in these genes were also reported to act as modifiers in the cancer process. ${ }^{[8]}$

Not as many genes are linked with FPTC as many with sporadic PTC. Some of the regions have been identified which can potentially harbour an FPTC gene: Chromosomal region 1q21 linked to FPTC with papillary renal neoplasia, ${ }^{[9]} 2 \mathrm{q} 21$ linked to familial NMTC type 1 syndrome $^{[10]}$ and the telomere abnormalities and chromosome fragility might display in FPTC family; ${ }^{[11]}$ specifically, familial NMTC and its relationship with familial MNG are recognized as distinct clinical entities, and the molecular pathophysiology of MNG and PTC is different, indeed, MNG1 is located at $14 \mathrm{q} ;{ }^{[12]}$ however, one study in a kindred with MNG and PTC suggested that $14 \mathrm{q} 32$ linked to a form of inherited MNG syndrome with a significant risk of progression to PTC. ${ }^{[13]}$ These findings are suggestive of a possible relationship between these genetic mutations and FPTC, but none of these are conclusive. Like any other familial conditions, the specific set of genetic mutations should be transmitted from one generation to the next to confirm familial nature of the condition. Liao et al. in their study concluded that in Chinese familial multinodular goitre (fMNG) with PTC, the risk allele homozygote of rs966423 (CC) at 2q35, rs2910164 (CC) at 5q24 and rs2439302 (GG) at 8 p12 could contribute to the $\mathrm{MNG}$ with PTC, while the other identified risk alleles for sporadic PTC or radiation-related PTC might not be involved. Furthermore, corresponding to the previous studies on the association between chromosome $14 \mathrm{q}$ and $\mathrm{fMNG}$ with PTC, their work approved that rs944289 but not rs116909374 at 14q13 locus might be associated with genetic predisposition to a Chinese family MNG with PTC. Although several different heterozygous alleles in the RET introns presented, the common BRAF and RET mutations were not susceptibility genetic events in this Chinese family. ${ }^{[14]}$ Clinicians have to be very careful about bringing up the word 'familial' with patients during their treatment. The most obvious subsequent question would be the risk of that condition in their children or siblings. Due to lack of evidence about genes linked to FPTC, the risk in subsequent generations cannot be quantified and no specific gene studies are routinely performed to look for any specific genes in high-risk patients so far. In this scenario, patients may be left confused and to 
some extent unnecessarily worried once they hear the word 'familial.' It is important to properly discuss with patients that close monitoring is the only way forward at the moment. Any of their relatives who may have got a thyroid condition should not take it lightly and should consult their endocrinologist for advice.

Clinicians should keep a lower threshold for invasive investigations like FNAC, for such patients who may fulfil the criteria of FPTC. The treatment plans for such patients should be discussed in multidisciplinary meetings and possibility of FPTC should be considered in highrisk patients. The aggressive nature of the condition may require more extensive surgery compared to sporadic forms of PTC. This has to be decided on individual basis, and at this stage, there is not enough evidence to suggest definitive treatment plan for all such patients. Familial nature of PTC remains a sensitive issue in clinical practice and due attention has to be paid to discuss it properly.

Multifocal papillary carcinoma is the most common biopsy finding in patients with FPTC. ${ }^{[7]}$ Biopsy of two of three sisters reported this pattern. Population-based studies have confirmed increased incidence of PTC in up to the third-degree relatives. Oakley et al. found that first-, second-, and third-degree relatives of PTC patients had a significantly higher risk of developing FPTC compared with general population used as controls. The first-degree relatives of the patients of FPTC were at a 5.4-fold increased risk $(P<10-15)$ of being diagnosed as having this cancer themselves. The second- and third-degree relatives had a 2.2-fold $(P<10-11)$ and 1.8-fold increased risk $(P<10-8)$, respectively. Siblings of patients were at highest risk (odds ratio, 6.8; $P<10-15) .{ }^{[4]}$ It is interesting to note that up to the third-degree relatives have higher incidences of FPTC. Involvement of up to the third-degree relatives makes genetic cause a very likely possibility; however, since the pattern of inheritance remains unclear, it is not possible so far to use it in clinical settings properly. It is important to remember that most population studies have recorded only numbers of patient with PTC and not recorded the type and nature of papillary cancer. It would be interesting to see if the nature aggressiveness of PTC could be recorded as well. Since other smaller data size studies have suggested, FPTC is a more aggressive form of disease; it would be interesting if further studies could differentiate PTC on the basis of aggressiveness of tumour.
This family showed some important clinical facts about inheritance of FPTC not reported in literature before. The age of diagnosis was very similar in all sisters. Two of them were diagnosed soon after their first presentation to specialist endocrinology unit following the first FNA while one sister was disease-free 10 years ago on FNA. FPTC generally presents at a younger age, but presentation at almost the same age is an interesting finding. All sisters lived in different parts of world and were exposed to different environmental conditions. Development of PTC in most of these sisters suggests a strong genetic influence in occurrence of FPTC. Population-based studies have also suggested a stronger genetic influence as the incidence is higher in up to the third-degree relatives; however, the incidence is not statistically significant in spouses of these patients. ${ }^{[4]}$

FPTC is usually more aggressive than its sporadic counterpart, but surgical treatment proved curative in all these patients of FPTC with no evidence of metastasis. This family emphasizes the strong genetic influence in the development of FPTC. Environmental factors may influence the development of FPTC, but it does not seem to be a strong factor in these patients. All these patients were diagnosed at very similar age, and it may be useful to screen relatives of patients with strong family history of FPTC at the age of diagnosis of the first patient.

\section{Conflict of Interest}

The authors declare that they have no conflict of interest.

\section{References}

1. Davies L, Welch HG. Increasing incidence of thyroid cancer in the United States, 1973-2002. JAMA 2006;295:2164-7.

2. Davies L, Welch HG. Epidemiology of head and neck cancer in the United States. Otolaryngol Head Neck Surg 2006;135:451-7.

3. Bonora E, Tallini G, Romeo G. Genetic predisposition to familial nonmedullary thyroid cancer: An update of molecular findings and state-of-the-art studies. J Oncol 2010;2010:385206.

4. Oakley GM, Curtin K, Pimentel R, et al. Establishing a familial basis for papillary thyroid carcinoma using the Utah population database. JAMA Otolaryngol Head Neck Surg 2013;139:1171-4.

5. Khan A, Smellie J, Nutting C, et al. Familial nonmedullary thyroid cancer: A review of the genetics. Thyroid 2010;20:795-801. 
6. Nosé V. Familial thyroid cancer: A review. Mod Pathol 2011;24 Suppl 2:S19-33.

7. Musholt TJ, Musholt PB, Petrich T, et al. Familial papillary thyroid carcinoma: Genetics, criteria for diagnosis, clinical features, and surgical treatment. World J Surg 2000;24:1409-17.

8. Nikiforov YE, Nikiforova MN. Molecular genetics and diagnosis of thyroid cancer. Nat Rev Endocrinol 2011;7:569-80.

9. Shifrin AL, Ogilvie JB, Stang MT, et al. Single nucleotide polymorphisms act as modifiers and correlate with the development of medullary and simultaneous medullary/ papillary thyroid carcinomas in 2 large, non-related families with the RET V804M proto-oncogene mutation. Surgery 2010;148:1274-80.

10. McKay JD, Lesueur F, Jonard L, et al. Localization of a susceptibility gene for familial nonmedullary thyroid carcinoma to chromosome $2 \mathrm{q} 21$. Am J Hum Genet 2001;69:440-6.

11. Cantara S, Pisu M, Frau DV, et al. Telomere abnormalities and chromosome fragility in patients affected by familial papillary thyroid cancer. J Clin Endocrinol Metab 2012;97:E1327-31.

12. Bignell GR, Canzian F, Shayeghi M, et al. Familial nontoxic multinodular thyroid goiter locus maps to chromosome $14 \mathrm{q}$ but does not account for familial nonmedullary thyroid cancer. Am J Hum Genet 1997;61:1123-30.

13. Bakhsh A, Kirov G, Gregory JW, et al. A new form of familial multi-nodular goitre with progression to differentiated thyroid cancer. Endocr Relat Cancer 2006;13:475-83.

14. Liao S, Song W, Liu Y, et al. Familial multinodular goiter syndrome with papillary thyroid carcinomas: Mutational analysis of the associated genes in 5 cases from 1 Chinese family. BMC Endocr Disord 2013;13:48. 